\title{
Carbamoylphosphate and natural auxotrophies in lactic acid bacteria
}

\author{
Françoise Bringel \\ Laboratoire de Microbiologie et de Génétique, Université Louis-Pasteur, CNRS URA, \\ $n^{\circ}$ D1481, 28, rue Goethe, 67083 Strasbourg, France
}

\begin{abstract}
Lactic acid bacteria require rich and complex media because they have multiple auxotrophies. A study of arginine and pyrimidine requirements of 113 homofermentative lactobacilli, mostly Lactobacillus plantarum and $L$. pentosus, showed that only $64 \%$ were protoptrophs for these compounds. $33 \%$ of the strains required arginine and uracil in aerobic growth conditions (but not in a carbon dioxide-enriched atmosphere), suggesting a deficiency of the common intermediate between the arginine and the pyrimidine metabolism: carbamoylphosphate (CP). No auxotrophs for the pyrimidines only were detected. Among the four auxotrophs for arginine only, three had no ornithine carbamoyltransferase (EC 2. 1.3.3), an enzyme that utilizes CP as a substrate. Thus, the CP metabolism appears to be a good tool to study homofermentative lactobacilli evolution and adaptation to their ecological niches. In this paper, CP metabolism and its variability within lactic acid bacteria will be presented. Depending of the species, $\mathrm{CP}$ is produced either from carbamoylphosphate synthetase (CPSase; EC 6. 3. 5. 5) from carbon dioxide, ATP and glutamine (L. plantarum), or from arginine catabolism via the arginine deiminase (ADI) pathway (Lactobacillus leichmannii). Among lactic acid bacteria, the ADI pathway is absent, partially or totally present with different regulation expressions. (C) Inra/Elsevier, Paris.
\end{abstract}

arginine / pyrimidine / carbamoylphosphate synthetase / arginine deiminase / auxotrophy

Résumé - Carbamyl phosphate et auxotrophies naturelles chez les bactéries lactiques. Les bactéries lactiques requièrent des milieux de culture très riches, du fait de leurs multiples auxotrophies. L'étude des besoins nutritionnels en arginine et en pyrimidines de 113 souches de lactobacilles homofermentaires, principalement des Lactobacillus plantarum et des L. pentosus, a montré que seulement $64 \%$ des souches étaient prototrophes pour ces composés ; $33 \%$ des souches nécessitent la présence d'arginine et d'uracile dans le milieu de culture en aérobiose (mais non en atmosphère enrichie en gaz carbonique) suggérant une déficience au niveau de l'intermédiaire commun entre ces deux métabolismes : le carbamyl phosphate (CP). Aucun auxotrophe pour les pyrimidines seules n'a été détecté et parmi les quatre souches auxotrophes pour l'arginine seul, trois souches sont dépourvues d'activité ornithine transcarbamylase (EC 2. 1. 3. 3) qui est une enzyme utilisant le CP comme substrat. Ainsi, le métabolisme du CP semble être un bon modèle d'étude de l'évolution et de l'adaptation des lactobacilles homofermentaires à leurs niches écologiques. Dans cet article nous faisons le point de l'état des connaissances et de la variabilité du métabolisme du CP chez les bactéries lactiques. Selon les espèces, le $\mathrm{CP}$ est soit synthétisé par la carbamyl phosphate synthétase (CPSase ; EC 6.3.5.5) à partir de gaz carbonique, d'ATP et de glutamine ( $L$, plantarum), soit provient du catabolisme de l'arginine par la voie de l'arginine désiminase (ADI) (Lactobacillus leichmannii). Au sein des bactéries lactiques, la voie de l'ADI peut être absente, partiellement ou totalement présente avec différentes régulations de son expression. (C) Inra/Elsevier, Paris.

arginine / pyrimidine / carbamyl phosphate synthétase / arginine désiminase / auxotrophie 


\section{INTRODUCTION}

Lactic acid bacteria have multiple auxotrophies. They require many amino acids, nucleobases, vitamins and cations. Among the different genera of lactic acid bacteria, Lactobacilli and leuconostocs have the most nutritional requirements. Having evolved in nutritionally rich environments, lactic acid bacteria have developed specific multiple auxotrophies in close association with their biotopes (fermented meats, salted fish, dairy products or fermented plants, mammalian cavities). These biosynthetic inabilities are characterized by the absence of functional activities (enzymes, regulators or transporters) which define specific enzymatic pathways. Several hypotheses may explain these auxotrophies. Either these proteins were never present in the microorganism, or were present but inactived by genetic lesions of the corresponding genes. The importance of these lesions was evaluated by mutagenesis. Morishita et al. tested the possibility of amino acid auxotrophy to prototrophy reversion in Lactobacilli (L. plantarum, $L$. casei, $L$. helveticus, L. acidophilus), Lactococcus lactis, Enterococcus faecium and Pediococcus acidilactici $[5,17]$. Since it was possible to reactivate prototrophy for certain amino acids, silent genes with minor alterations (such as punctual mutations) were present. For instance, a single mutation in the ARN polymerase of E. faecium mutants rendered protrophy for several amino acids simultaneously [5]. For other amino acids, no prototrophs were obtained, suggesting major lesions (such as deletions) or total absence of the corresponding genes. In Le. lactis, small deletions found in the leucine biosynthesis structural genes did not alter regulation and transcription of these genes [8]. In the Le. lactis histidine biosynthetic genes, mutations in the promoter, frameshift mutations in three structural genes and insertion of two genes unrelated to the bio- synthetic pathway were found [6]. The nutritional requirements for arginine and the pyrimidines of more than 100 homofermentative Lactobacilli isolated from various environments, were tested in our laboratory; $64 \%$ of the Lactobacilli were prototrophs for these compounds, $3 \%$ auxotrophs for arginine only and 33\% auxotrophs for arginine and uracil. The latter are conditional auxotrophs since they require these compounds only in aerobic conditions. The requirement of both arginine and pyrimidines suggests a deficiency in the carbamoylphosphate (CP) metabolism. Indeed, CP is a common intermediate in both the pyrimidine biosynthesis (the first steps) and arginine metabolism (the fifth step in biosynthesis and the two last steps of catabolism via the arginine deiminase pathway) (figure 1 ). In this paper, lactic acid bacteria CP metabolism's natural auxotrophies and its related pathways will be reviewed.

\section{CARBAMOYLPHOSPHATE (CP) IN THE ARGININE CATABOLISM VIA THE ARGININE DEIMINASE PATHWAY (ADI)}

Only certain lactic acid bacteria degrade arginine. The ADI pathway, also called arginine dihydrolase, provides energy by a substrate phosphorylation process with the formation of one mol of ATP per mol of arginine consumed. However, arginine serves as sole energy and carbon source for a limited number of lactic acid bacteria (Lactobacillus confusus, certain Lactococcus lactis and Enterococcus) [13]. In Enterococci, this pathway helps to protect microorganisms against acid damage by a rise in $\mathrm{pH}$ associated with ammonia production [14]. Arginine is degraded into ammonia, carbon dioxide, ATP and ornithine in three successive enzymatic steps. Arginine deiminase (EC 3.5.3.6) degrades arginine into citrulline and ammonia. 


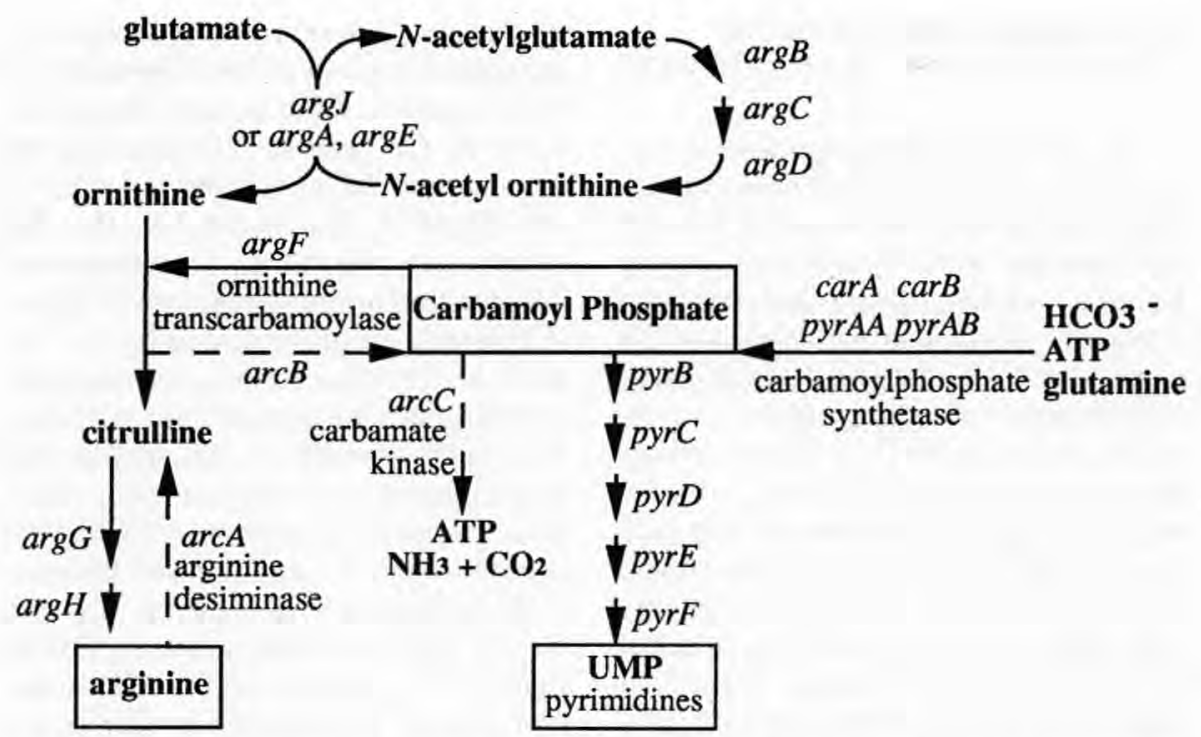

Figure 1. Carbamoylphosphate, an intermediate in the pyrimidine and arginine metabolism. Dashed arrows indicate the three steps involved in arginine catabolism via the arginine deiminase pathway (arginine deiminase, catabolic ornithine carbamoyltransferase and carbamate kinase). Solid arrows indicate the biosynthetic steps.

Figure 1. Le carbamyl phosphate, un intermédiaire du métabolisme des pyrimidines et de l'arginine. Les flèches en pointillés indiquent les trois étapes impliquées dans le catabolisme de l'arginine par la voie de l'arginine désiminase (arginine désiminase, ornithine transcarbamylase catabolique et la carbamate kinase). Les flèches continues indiquent les étapes de biosynthèse.

Catabolic ornithine carbamoyltransferase (EC 2.1.3.3) cleaves citrulline in $\mathrm{CP}$ and ornithine. Carbamate kinase (EC 2.7.2.2) produces ATP, ammonia and carbon dioxide if CP is dephosphorylated (figure 1). When this pathway is present in streptococci, an ornithine/arginine antiporter expels ornithine out of the cell in exchange for extracellular arginine, using the ornithine and arginine concentration gradients as the sole energy source [18]. The ADI pathway is present in lactococci ( $L c$. lactis subsp. lactis), Lactobacilli (L. brevis, L. buchneri, L. confusus, L. leichmannii, L. sake), Carnobacterium, certain strains of Streptococcus thermophilus, Enterococcus faecalis, Oenococcus oeni and Pediococcus (P. acidilactici, P. pentosaceus). The ADI pathway is absent in
Lc. lactis subsp. cremoris, and some Lactobacilli (L. acidophilus, L. bulgaricus, L. casei, L. delbrueckii, L. helveticus, L. paraplantarum, L. pentosus, L. plantarum) $[3,4,10,12,13,16]$. Arginine induces the ADI pathway at different levels in various organisms. In Lc. lactis, arginine triggers the expression of arginine deiminase and catabolic ornithine transcarbamoylase (as well as the carbamate kinase in E. faecalis) but has no effect on carbamate kinase which is constitutive [2]. In general, the enzymes of the ADI pathway are active in bacteria grown with limited energy resources [3]. Thus, glucose seems to repress the ADI pathway by catabolic repression (cyclic AMP which signals an energy deficiency antagonizes glucose repression) [18]. 


\section{NATURAL DEFICIENCIES FOUND IN THE ADI PATHWAY}

The lack of arginine catabolism of certain lactic acid bacteria has been correlated to total absence or partial presence of ADI pathway enzymatic activities. Among Oenococcus oeni strains isolated from wine, $77 \%$ degrade arginine by this ADI pathway and $23 \%$ have no detectable activity for one of the three enzymes involved in the pathway [12]. These authors also isolated a strain of $L$. brevis from wine having no arginine deiminase activity [12]. Natural variants of $E$. faecalis were found to be unable to use arginine as an energy and carbon source because they lacked at least one of the three enzymes of the ADI pathway or the ornithine/arginine antiporter, or had weak activities for the ADI pathway enzymes [3].

Even though Lc. lactis subsp. cremoris is unable to degrade arginine since it lacks arginine deiminase or the ornithine/arginine antiporter or, in some strains, even ornithine transcarbamoylase, a mutant harboring arginine deiminase and ornithine transcarbamoylase was found [2]. This revertant suggests punctual mutations and not the total absence of the ADI genes in some strains of $L c$. lactis subsp. cremoris.

\section{CP, A PRECURSOR OF THE PYRIMIDINES AND ARGININE}

Little is known about the genetics of these pathways in lactic acid bacteria. Two $\mathrm{CP}$ production pathways have been described. In the first, the ADI pathway produces $\mathrm{CP}$ which is subsequently used for pyrimidine biosynthesis. This situation is unusual for procaryotes and has clearly been demonstrated in an arginine auxotrophic strain, L. leichmannii [9]. In the second and more common pathway, CP is synthesized from ATP, glutamine and bicarbonate by the carbamoylphosphate synthetase (CPSase) which is an allosteric enzyme composed of two sub-units [3]. In Escherichia coli, only one CPSase provides the $\mathrm{CP}$ necessary for the arginine and the pyrimidine biosynthesis. On the other hand, $L$. plantarum (like Bacillus subtilis), has two independently regulated CPSases: an arginine-repressed CPSase (CPSaseArginine encoded by the car genes [1]; personal communication) and a pyrimidine-regulated CPSase (CPSasePyrimidines encoded by the pyrAApyrAB genes present in the biosynthetic pyrimidine operon) [7]. L. plantarum CCM 1904 is prototrophic for arginine and the pyrimidines. However, in presence of uracil, the pyr operon is inhibited and CPSasePyrimidines is absent so that CP formation can only be catalysed by CPSaseArginine. In fact, uracil inhibits $L$. plantarum growth in aerobiosis but not in anaerobiosis, or when grown in an atmosphere enriched with $4 \%$ on carbon dioxide. This conditional sensitivity to uracil suggests that the $L$. plantarum CPSaseArginine is inhibited by oxygen [15] or has a low affinity for one of its substrates, bicarbonate which is the dissolved form of carbon dioxide. Another unknown mechanism may also be involved. In E. faecalis, Li et al. [11] demonstrated the occurrence of a CPSasePyrimidines encoded by genes present in a pyr operon. This organism is peculiar since it has two carbamate kinases [19]. One carbamate kinase is part of the ADI pathway and the other belongs to an analogous pathway, the agmatine deiminase pathway. Agmatine is catabolized in ATP, carbon dioxide and ammonia in three steps catalysed by the agmatine deiminase, the putrescine carbamoyltransferase and the carbamate kinase. Glucose and arginine repress the agmatine deiminase pathway. An ADI-deficient mutant can grow if agmatine is provided in the medium [19]. Whether CP produced from agmatine catabolism can be directed towards the pyrimidine and arginine biosynthesis or if a CPSaseArginine is pre- 
sent in this organism is not known. The agmatine deiminase pathway has not been described in other lactic acid bacteria [3].

\section{NATURAL DEFICIENCIES FOUND IN THE CP METABOLISM}

A strain deficient in the CP metabolism is auxotrophic for arginine and/or the pyrimidines. In our laboratory, the nutritional requirements for arginine and uracil have been studied for 113 homofermentative lactobacilli, mostly L. plantarum and $L$. pentosus, but also $L$. paracasei and $L$. paraplantarum. These lactobacilli were isolated from dairy products, fermented plants (silage, green olives, cucumber, cabbage, cassava, corn, millet, beer) or from human origin (saliva, feces). Growth was tested at $30^{\circ} \mathrm{C}$ in standard atmosphere (aerobiosis) or in an atmosphere enriched with $4 \%$ carbon dioxide (water-jacketed incubator $\mathrm{CH} / \mathrm{P}$, Forma Scientific, Inc) on agar-plates of defined media DLA [1] supplemented or not with uracil, arginine or an arginine precursor such as ornithine or citrulline, at a final concentration of $50 \mu \mathrm{g} / \mathrm{mL}$. $64 \%$ of the Lactobacilli grew on the DLA medium and were therefore arginine and uracil prototrophs. $3 \%$ of the lactobacilli (four strains) were auxotrophs for arginine and citrulline suggesting that the genetic lesion was not in the $\arg G H$ genes involved at the last steps of arginine biosynthesis (see figure I). Adding ornithine, which is the direct precursor of citrulline, to the DLA medium did not restore prototrophy in three Lactobacilli ( $L$. pentosus CNRZ 1555 , L. paracasei NCIMB 3254 and CST 19027). This observation was confirmed by the absence of detectable ornithine transcarbamoylase which catalyses the formation of citrulline from ornithine and $\mathrm{CP}$, incriminating a genetic lesion in $\arg F$ coding for the ornithine transcarbamoylase in these strains. On the other hand, the addition of ornithine but not of citrulline in the DLA media restored growth of $L$. pentosus CNRZ 1245. Thus, CNRZ 1245 is an arginine auxotroph by lacking an enzyme encoded by the $\operatorname{argBCDJ}(A E)$ cluster (figure 1 ). The other tested lactobacilli were conditional auxotrophs since in order to grow on the defined DLA medium, they required arginine (or citrulline) and uracil in aerobiosis but not in an atmosphere enriched with $4 \%$ of carbon dioxide. Bicarbonate (dissolved carbon dioxide) is a substrate of the CPSase catalysing CP formation with glutamine and ATP. The double requirement for uracil and arginine suggests that the conditional auxotrophs are deficient in the $\mathrm{CP}$ metabolism. The molecular target of the lesions need to be defined by enzymatic activity assays and in vivo complementation tests with functional genes cloned from prototrophic Lactobacilli. Among the conditional auxotrophs, some strains are sensitive to uracil ( $L$. pentosus CNRZ 1573 and $L$. plantarum JCL 1279 ) or to arginine (L. pentosus CNRZ 1547 ) since they did not grow on DLA medium in a carbon dioxide-enriched atmosphere in presence of uracil or arginine respectively. As a conclusion, the genetic alterations found in CP biosynthesis of homofermentative lactobacilli affect either catalytic and regulatory sites of enzymes implied in this metabolism or regulation elements. The extent of the genetic lesions varies from strain to strain even within a same species. For instance, no revertant could be obtained from the auxotroph $L$. pentosus CNRZ 1573. On the contrary, L. pentosus CNRZ 1547 lost its arginine sensitivity at a frequency of 10-7 and even arginine and uracil prototrophs were isolated.

\section{CONCLUSION}

A preliminary study of the arginine and pyrimidine nutritional requirements of homofermentative Lactobacilli showed 
that not all the steps involved in these metabolisms are subject to genetic degeneration. In fact, no auxotrophs for the pyrimidines only were detected. $33 \%$ of the tested auxotrophs are conditional auxotrophs for both arginine and the pyrimidines and seem to have deficient CP metabolism. Among the four Lactobacilli auxotrophs for only arginine, three were deficient for the ornithine transcarbamoylase $(\arg F)$ directly using $\mathrm{CP}$ as a substrate. CP is an important metabolic bottleneck, which appears as a privileged target for Lactobacilli adaptation to their ecological niches. Moreover, the cloning and sequencing of the arginine biosynthetic cluster of a prototrophic $L$. plantarun revealed the presence of carA (coding for the small sub-unit of the CPSaseArginine) transcribed divergently from the $\mathrm{arg}$ genes. This organization is different from the other Gram-positive arg clusters studied so far [1]. This peculiarity in gene organization reinforces the key role of $\mathrm{CP}$ in the metabolism and evolution of lactic acid bacteria. CP production is controlled at different levels: transcription (attenuation control by the PyrR regulator of the L. plantarum pyr operon [7]; repression of the $L$. plantarum arg cluster [1]) and directly on the enzymes by allosteric regulation [3]. Thus, lactic acid bacteria have many possibilities to adapt their CP metabolism to specific growing conditions. Depending on the species (L. leichmannii, L. plantarum, E. faecalis), different strategies for CP synthesis have evolved. For instance, we identified the stimulatory effect of carbon dioxide on the growth of the homofermentative Lactobacilli conditional auxotrophs. In the natural fermentation process, carbon dioxide is commonly encountered because it is produced by heterofermentative lactic acid bacteria. Homofermentative lactic acid bacteria do not produce carbon dioxide massively since lactic acid is their main end product. The growth of the conditional auxotrophs would be facilitated in eco- logical niches containing bacteria which produce carbon dioxide when the stock of pyrimidines and arginine is limited. Several hypotheses could explain the mode of action of carbon dioxide and bicarbonate on the growth of the conditional auxotrophs for arginine and the pyrimidines. The conditional auxotrophs may have an insufficient CPSase activity because the CPSase has a reduced affinity for its substrate, bicarbonate. If the culture conditions provide plenty of bicarbonate (by growing the cells in an atmosphere enriched with carbon dioxide), CPSase activity would be sufficient to allow cell growth. Another possibility is the presence of a catabolic carbamate kinase which degrades $\mathrm{CP}$ into carbon dioxide, ammonia and ATP. Although L. plantarum, L. pentosus and $L$. paraplantarum lack the two initial steps of the ADI pathway $[4,13]$, in the last step of the pathway, carbamate kinase may be present. Since carbon dioxide is a product of carbamate kinase, high concentrations of carbon dioxide could lower the carbamate kinase activity so that more $\mathrm{CP}$ would be present for the arginine and the pyrimidine biosynthesis.

The screening for natural auxotrophs having $\mathrm{CP}$ metabolism deficiencies showed that certain lactic acid bacteria have genetic lesions which allow for more or less permanent adaptation of their $\mathrm{CP}$ metabolism to growth conditions (possibility to revert to prototrophy; inhibition by oxygen and stimulation by carbon dioxide).

\section{REFERENCES}

[1] Bringel F., Frey L., Boivin S, Hubert J.C., Arginine biosynthesis and regulation in $\mathrm{Lac}$ tobacillus plantarum: the carA and the $\arg C$. $J B D F$ cluster are divergently transcribed, J. Bacteriol. 179 (1997) 2697-2706.

[2] Crow V.L., Thomas T.D., Arginine metabolism in lactic streplococci, J. Bacteriol. 150 (1982) 1024-1032. 
[3] Cunin R., Glansdorff N., Piérard A., Stalon V., Biosynthesis and metabolism of arginine in bacteria, Microbiol. Rev. 50 (1986) 314-352.

[4] Curk M.C., Hubert J.C., Bringel F., Lactobacillus paraplantarum sp. nov., a new species related to Lactobacillus plantarum, Int. J. Syst. Bacteriol. 46 (1996) 595-598.

[5] Deguchi Y., Morishita T., Nutritional requirements in multiple auxotrophic lactic acid bacteria: genetic lesions affecting amino acid biosynthesis pathways in Lactococcus lactis, Enterococcus faecium, and Pediococcus acidilactici, Biosci. Biotech. Biochem. 56 (1992) 913-918.

[6] Delorme C., Godon J.J., Ehrlich S.D., Renault P., Gene inactivation in Lactococcus lactis: histidine biosynthesis, J. Bacteriol. 175 (1993) 4391-4399.

[7] Elagöz A., Abdi A., Hubert J.C., Kammerer B., Structure and organisation of the pyrimidine biosynthesis pathway genes in Lactobacillus plantarum: a PCR strategy for sequencing without cloning, Gene 182 (1996) 37-43.

[8] Godon J.J., Delorme C., Bardowski J., Chopin M.C., Ehrlich S.D., Renault P., Gene inactivation in Lactococcus lactis: branched-chain amino acid biosynthesis, J. Bacteriol. 175 (1993) 4383-4390.

[9] Hutson J.K., Downing M., Pyrimidine biosynthesis in Lactobacillus leichmannii, J. Bacteriol. 96 (1968) 1249-1254.

[10] Leisner J.J., Tidemand J., Larsen L.M., Catabolism of arginine by Carnobacterium spp. isolated from vacum-packed sugar-salted fish, Curr. Microbiol. 29 (1994) 95-99.

[11] Li X.,Weinstock G.M., Murray B.E., Generation of auxotrophic mutants of Enteroccus faecalis, J. Bacteriol. 177 (1995) 6866-6873.
[12] Liu S.Q., Pritchard G.G., Hardman M.J., Pilone G.J., Occurrence of arginine deiminase pathway enzymes in arginine catabolism by wine lactic acid bacteria, Appl. Environ Microbiol. 61 (1995) 310-316.

[13] Manca de Nadra M.C., Pesce de Ruiz Holgado A.A., Oliver G., Arginine dihydrolase activity in lactic acid bacteria, Milchwissenschaft 37 (1982) 669-670.

[14] Marquis R.E., Bender G.R., Murray D.R., Wong A., Arginine deiminase system and bacterial adaptation to acid environments, Appl. Environ. Microbiol. 53 (1987) 198-200.

[15] Masson A., Étude génétique, physiologique et biochimique du métabolisme des pyrimidines chez Lactobacillus plantarum, Thèse de Doctorat d'Université Lowuis-Pasteur de Strasbourg, France (1992).

[16] Montel M.C., Champomier M.C., Arginine catabolism in Lactobacillus sake isolated from meat, App. Environ. Microbiol. 53 (1987) 2683-2685.

[17] Morishita T., Deguchi Y., Yajima M., Sakurai T., Yura T., Multiple nutritional requirements of lactobacilli: genetic lesions affecting amino acid biosynthetic pathways, J. Bacteriol. 148 (1981) 64-71.

[18] Poolman B., Driessen A.J.M., Konings W.N., Regulation of arginine-ornithine exchange and the arginine deiminase pathway in Streptococcus lactis, J. Bacteriol. 169 (1987) 5597-5604.

[19] Simon J.P., Stalon V., Enzymes of agmatine degradation and the control of their synthesis in Streptococcus faecalis, J. Bacteriol. 152 (1982) 676-681. 